\title{
A Comparison of Surface-Modeling Techniques $^{\dagger}$
}

\author{
Kubilay Sertel ANd LeVent Gürel* \\ Department of Electrical and Electronics Engineering \\ BILKENT UNIVERSITY \\ BilKENT, ANKARA, TURKEY \\ (lgureløee.bilkent.edu.tr)
}

\section{Introduction}

Solution techniques based on surface integral equations (SIEs) are widely used in computational electromagnetics. Formulations employing SIEs express the unknown function on the defining surface of the problem geometry. Thus, both the surface and the unknown function defined on it have to be accurately represented in the solution algorithm. However, real-life problems usually involve arbitrary geometries with curved surfaces, which require either exact or higher-order geometry models. In this work, the dependence of the accuracy of the solution on the geometry modeling is investigated. The use of different basis functions to represent the unknown functions on curved surfaces will also be mentioned.

\section{Surface Models}

Several different schemes exist for modeling the geometries of problems that are formulated using SIEs. In the following we will briefly mention the surface-modeling techniques that we are investigating.

\subsection{Exact Models}

Occasionally, exact geometry model of a scatterer may exist. For instance, a body composed of spherical, cylindrical, conical, polynomial, and flat surfaces can be exactly represented. In this summary, we will consider a sphere as a sample problem, for which an exact model exists.

\subsection{Polynomial Surfaces}

In most real-life problems, the scatterer is so complicated that it cannot be exactly represented. One is forced to use approximations. The simplest scheme is approximating

\footnotetext{
'This work was supported in part by NATO's Scientific Affairs Division in the framework of the Science for Stability Programme and in part by the Scientific and Technical Research Council of Turkey (TUBITAK) under contract EEEAG-163.

0-7803-4178-3/97/\$10.00 ( 1997 IEEE
} 
the scatterer by polynomial patches. In this work, we are interested in the following polynomial surface descriptions:

\section{- Flat Triangulation}

In this modeling method, the geometry of the scatterer is approximated by a mesh of connected flat triangular patches. This is a powerful method for modeling arbitrarily shaped scatterers due to its flexibility. Although it is so powerful, accurate representation of an arbitrary surface requires a large number of triangular patches. Since the basis functions used in the discretization of the SIE are defined on these patches, the size of the problem is directly proportional to the number of triangular patches used.

\section{- Quadratic (Triangular) Patches}

A better approximation scheme for functions is the piecewise quadratic polynomial fitting or interpolation. A surface of arbitrary shape can be represented by a mesh of connected quadratic subsurfaces of the form

$$
\mathbf{r}(s, t)=\mathbf{a} s^{2}+\mathbf{b} t^{2}+\mathbf{c} s t+\mathbf{d} s+\mathbf{e} t+\mathbf{f},
$$

each of which is uniquely determined by 6 discrete points in space.

\section{- Biquadratic Patches}

Biquadratic patches are similar to quadratic patches, however, they are defined as Cartesian product surfaces

$$
\mathbf{r}(s, t)=\sum_{m=1}^{3} \sum_{n=1}^{3} \mathbf{C}_{m n}^{(p)} s^{m-1} t^{n-1}
$$

each of which is uniquely determined by 9 discrete points in space.

\subsection{Non-Uniform Rational B-Spline (NURBS) Surfaces}

If we examine the available computer-aided graphical design (CAGD) tools for bodies that are fabricated by using automated machining processes, we can conclude that nearly all of them are based on NURBS meshes. A NURBS surface is a B-Spline element and consists of a set of smoothly connected Bézier patches. A rational Bézier surface is defined as the ratio of two polynomials. The surface is described by a set vertices forming a mesh which is called the defining mesh. The surface follows the shape of the defining mesh, and it does not pass through any interior nodes of the mesh.

\section{Basis Functions}

In this work, piecewise linear functions on rectangular and triangular domains are used. On curved surfaces, these basis functions are defined as the generalizations of the well known rooftop (RT) [1-4] and triangular functions due to Rao, Wilton, and Glisson (RWG) [5]. On geometries represented with flat triangles, the usual "flat" RWG basis functions are used [6]. 


\section{Results}

Consider the problem of plane-wave scattering by a perfect-electric-conductor (PEC) sphere of radius $k a=0.4 \pi$. The incident plane-wave is given by $\mathbf{E}^{i}=\hat{x} e^{i k z}$. Figures $1-3$ show the far-zone $E_{\theta}$ on the $\phi=0$ cut and the far-zone $E_{\phi}$ on the $\phi=\frac{\pi}{2}$ cut. For comparison purposes, these results are obtained using the following four solution techniques:

1. Mie-series technique [7] is used to obtain a closed-form reference solution which is plotted using a solid line in Figs. 1-3. This solution satisfies the boundary condition on the sphere with an accuracy of $10^{-3}$.

2. A SIE technique is used with an exact geometry model of the sphere and the curved rooftop basis functions. These results are represented by " $x$ " symbols in Figs. 1-3.

3. Results represented by "t" symbols in Figs. 1-3 are obtained from a SIE solution with an exact model of the sphere and the curved RWG basis functions.

4. Results obtained using a flat triangular model of the sphere and the "flat" RWG basis functions in conjunction with a SIE solver are depicted by " $\oplus$ " symbols in Figs. 1-3.
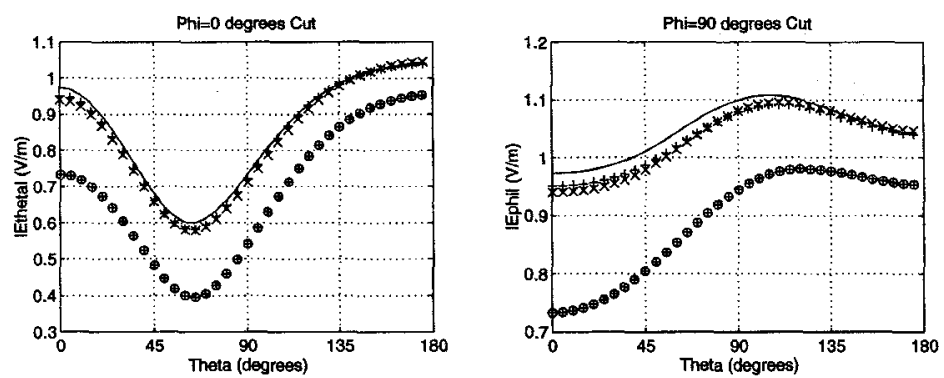

Figure 1: Comparison of far-zone fields obtained by Mie series (-), 54 curved rooftops $(\times), 54$ curved RWGs $(+)$, and 54 Hat RWGs $(\oplus)$, as explained in the text.
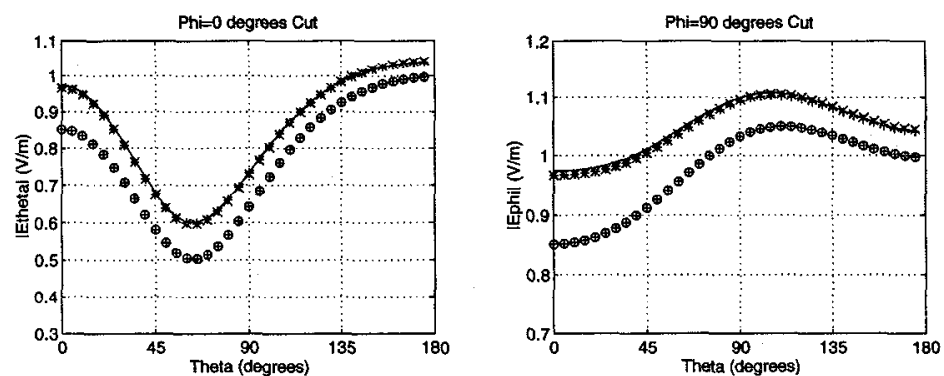

Figure 2: Comparison of far-zone fields obtained by Mie series $(-), 169$ curved rooftops $(\times), 144$ curved RWGs $(+)$, and 144 flat RWGs $(\oplus)$, as explained in the text. 
Different discretizations of both the exact and the flat-triangulation models are used in Figs. 1-3, where an approximately equal number of basis functions are used in each plot for comparison purposes. In all these results, we notice that the SIE solutions employing an exact model of the sphere are more accurate than those employing flat-triangulation models. Also, comparing Figs. 1 and 3, we observe that a much finer triangulation with 483 basis functions is required for flat triangles to obtain nearly the same accuracy obtained by using an exact geometry model with 54 RT or RWG basis functions.
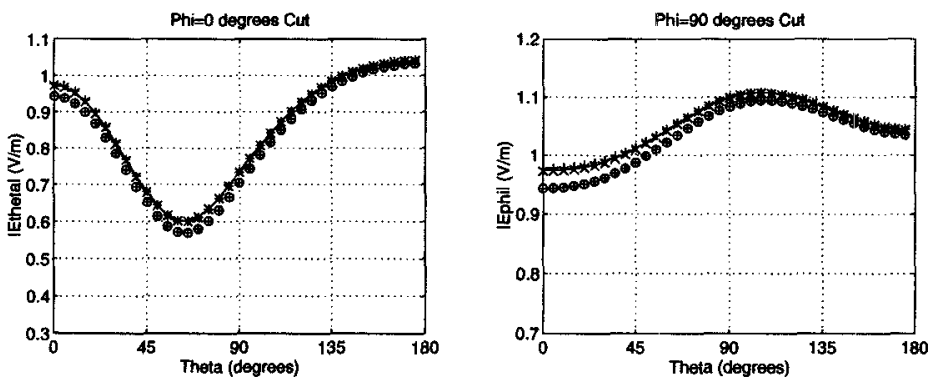

Figure 3: Comparison of far-zone fields obtained by Mie series (-), 483 curved rooftops $(\times), 480$ curved RWGs $(+)$, and 480 flat RWGs $(\oplus)$, as explained in the text.

\section{Conclusions}

By using exact and flat-triangulation models for a sphere, it is shown that accurate surface models increase the accuracy of the solutions. Alternatively, for a required solution accuracy, the problem size can be significantly reduced by using better geometry models for the scatterers. We are currently investigating the accuracy of the quadratic and NURBS surface models. It is observed that the type of the basis function is less effective than the quality of the geometry model in determining the accuracy of the solution.

\section{References}

[1] A. W. Glisson and D. R. Wilton, "Simple and efficient numerical methods for problems of electromagnetic radiation and scattering from surfaces," IEEE Trans. Antennas Propagat., vol. AP-28, no. 5 , pp. 593-603, Sept. 1980

[2] B. J. Rubin and S. Daijavad, "Radiation and scattering from structures involving finite-size dielectric regions," IEEE Trans. Antennas Propagat., vol. AP-38, pp. 1863-1873, Nov. 1990.

[3] J. M. Song and W. C. Chew, "Moment method solutions using parametric geometry," J. Electromagnetic Waves and Applications, vol. 9, no. 1/2, pp. 71-83, 1995.

[4] L. Valle, F. Rivas, and M. F. Cátedra, "Combining the Moment Method with Geometrical Modeling by NURBS Surfaces and Bézier Patches," IEEE Trans. Antennas Propagat., vol. AP-38, pp. 373381, Mar. 1994.

[5] S. Wandzura, "Electric Current Basis Functions for Curved Surfaces," Electromagnetics, vol.. 12, pp. 77-91, 1992.

[6] S. M. Rao, D. R. Wilton, and A. W. Glisson, "Electromagnetic scattering by surfaces of arbitrary shape," IEEE Trans. Antennas Propagat., vol. AP-30, pp. 409-418, May 1982.

[7] C. A. Balanis, Advanced Engineering Electromagnetics. New York: Wiley, 1989. 